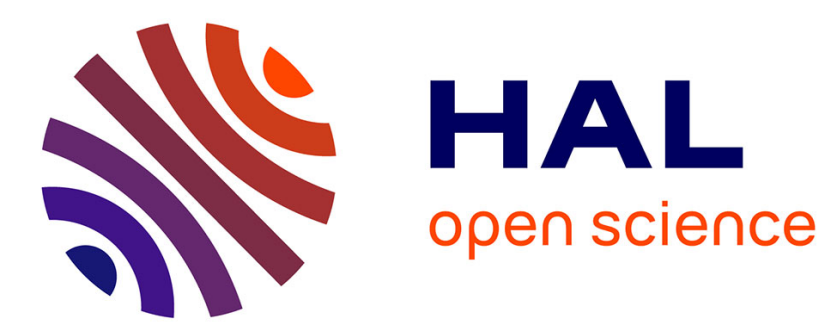

\title{
Flash sintering of dielectric nanoparticles as a percolation phenomenon through a softened film
}

Rachman Chaim, Geoffroy Chevallier, Alicia Weibel, Claude Estournès

\section{To cite this version:}

Rachman Chaim, Geoffroy Chevallier, Alicia Weibel, Claude Estournès. Flash sintering of dielectric nanoparticles as a percolation phenomenon through a softened film. Journal of Applied Physics, 2017, 121 (14), pp.45103. 10.1063/1.4980853 . hal-01540058

\section{HAL Id: hal-01540058 \\ https://hal.science/hal-01540058}

Submitted on 15 Jun 2017

HAL is a multi-disciplinary open access archive for the deposit and dissemination of scientific research documents, whether they are published or not. The documents may come from teaching and research institutions in France or abroad, or from public or private research centers.
L'archive ouverte pluridisciplinaire HAL, est destinée au dépôt et à la diffusion de documents scientifiques de niveau recherche, publiés ou non, émanant des établissements d'enseignement et de recherche français ou étrangers, des laboratoires publics ou privés. 


\section{Open Archive TOULOUSE Archive Ouverte (OATAO)}

OATAO is an open access repository that collects the work of Toulouse researchers and makes it freely available over the web where possible.

This is an author-deposited version published in : http://oatao.univ-toulouse.fr/ Eprints ID : 17901

To link to this article : DOI:10.1063/1.4980853

URL : $\underline{\text { http://dx.doi.org/10.1063/1.4980853 }}$

To cite this version : Chaim, Rachman and Chevallier, Geoffroy and Weibel, Alicia and Estournès, Claude Flash sintering of dielectric nanoparticles as a percolation phenomenon through a softened film. (2017) Journal of Applied Physics, vol. 121 ( $\mathrm{n}^{\circ}$ 14). pp. 145103/1145103/5. ISSN 0021-8979

Any correspondence concerning this service should be sent to the repository administrator: staff-oatao@,listes-diff.inp-toulouse.fr 


\title{
Flash sintering of dielectric nanoparticles as a percolation phenomenon through a softened film
}

\author{
Rachman Chaim, ${ }^{1, a)}$ Geoffroy Chevallier, ${ }^{2, b)}$ Alicia Weibel, ${ }^{2, c)}$ and Claude Estournès ${ }^{2, d)}$ \\ ${ }^{1}$ Department of Materials Science and Engineering, Technion - Israel Institute of Technology, Haifa 32000, \\ Israel \\ ${ }^{2}$ Université de Toulouse, CIRIMAT, CNRS INPT UPS, Université Paul-Sabatier, 118 route de Narbonne, \\ F-31062 Toulouse cedex 9, France
}

\begin{abstract}
Recent work [Biesuz et al., J. Appl. Phys. 120, 145107 (2016)] showed analogies between the flash sintering and dielectric breakdown in $\alpha$-aluminas pre-sintered to different densities. Here, we show that flash sintering of dielectric nanoparticles can be described as a universal behavior by the percolation model. The electrical system is composed of particles and their contact point resistances, the latter softened first due to preferred local Joule heating and thermal runaway during the flash. Local softening has a hierarchical and invasive nature and propagates between the electrodes. The flash event signals the percolation threshold by invasive nature of the softened layer at the particle surfaces. Rapid densification is associated with local particle rearrangements due to attractive capillary forces induced by the softened film at the particle contacts. Flash sintering is a critical phenomenon with a self-organizing character. The experimental electric conductivity results from flash sintering are in full agreement with those calculated from the percolation model.

[http://dx.doi.org/10.1063/1.4980853]
\end{abstract}

\section{INTRODUCTION}

Rapid sintering and densification of dielectric powder compacts by flash sintering (FS) and spark plasma sintering (SPS) are attractive and innovative. Electric field or current is applied to the dielectric powder compact, while heated within a furnace. Many works modeled the SPS system to reveal its corresponding densification mechanisms. The high densification rates observed during SPS were related to the formation of local spark and plasma due to the particle surface discharges, ${ }^{1,2}$ materials jets, ${ }^{3,4}$ softening-assisted particle rearrangement and densification, ${ }^{5}$ as well as local plastic deformation. ${ }^{6,7}$ However, many aspects of the densification mechanisms during FS are still unclear and subject to scholarly debate. The different atomistic mechanisms suggested for the rapid densification during the flash sintering were presented in the last comprehensive review on flash sintering. ${ }^{8}$ Two different views of the densification mechanisms in FS exist, which refer to processes either in the bulk or at the particle surfaces and grain boundaries. Cologna et al. ${ }^{9}$ investigated the flash sintering of undoped and $\mathrm{MgO}$-doped alumina, and attempts to flash-sinter undoped alumina at $1400^{\circ} \mathrm{C}$ and $1000 \mathrm{~V} \cdot \mathrm{cm}^{-1}$ led to observation of sparks in the specimen. Furthermore, the application of cyclic current-voltage during FS of MgO-doped alumina at $1300^{\circ} \mathrm{C}$ revealed shrinking hysteresis curves, indicating that interfaces play a strong role during the flash sintering. These investigations confirm the interfacial nature

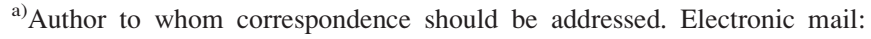
rchaim@technion.ac.il

b)chevallier@chimie.ups-tlse.fr

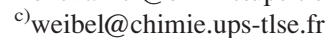

d)estournes@chimie.ups-tlse.fr
}

of the flash sintering process. These findings are in agreement with the recent flash sintering analysis by Biesuz et al. ${ }^{10}$ on partially sintered alumina at the similar FS conditions, where the flash sintering mechanism at the early stages of the flash was related to the "pre-breakdown" dielectric behavior.

The main drawback in understanding the physical processes and densification mechanisms in FS is due to the erratic expectation for linear electrical behavior (i.e., Ohm law) in granular dielectrics with the increase in temperature/ electric current/voltage. The electrical conductance through granular systems, comprised of various resistors, is a wellstudied problem, and its percolative nature with non-linear behavior has been verified. ${ }^{11,12}$ In addition, many of the granular systems exhibit fractal character, which further deviates their electric response from linearity. In their recent work, Biesuz et al. ${ }^{10}$ used $\alpha$-aluminas pre-sintered to different relative densities as compacts for flash sintering. They found a correlation between the activation of flash sintering and the compact porosity; dense specimens did not flashsinter even at very high electric fields. Using the porosity dependence of the electric conductivity, they related the compact non-ohmic behavior to the "pre-breakdown" phenomenon. Flash sintering of alumina, containing different amounts of a glassy phase, revealed enhanced sintering, where the electric current mainly passed through the liquid second-phase. ${ }^{13}$ Here, we show the thermal-electrical process characteristics in the dielectric nanoparticle compact subjected to flash sintering, which leads to a sudden increase in electric conductivity in accordance with the invasive percolation system. Rapid densification during the flash sintering is therefore a critical phenomenon with the selforganizing character. 


\section{THE PERCOLATION MODEL}

A powder compact made of non-conducting dielectric particles, as three-dimensional (3D) random media, consists of two types of resistors, and one type of capacitor, connected in parallel and in series. The two resistors represent the resistance of the particles and of the particle contacts (bonds), both varying with the particle size, within the frame of the percolation theory terminologies. The capacitors represent the gaps between the non-contacting particles, by which their capacitance also varies with the particle size and gap distance in this percolative system. In a previous work, ${ }^{14}$ this model was used for evaluation of the spark and plasma formation at the particle gaps during spark plasma sintering, hence the significance of the capacitor for surface charging and discharging. The loose powder compact (relative density, $\mathrm{RD}<50 \%$ ) in SPS conditions subjected to an applied pressure and to low electric fields (few $\mathrm{V} \cdot \mathrm{cm}^{-1}$ ). In contrast, the flash sintered specimens are green compacted powders (RD $\sim 60 \%$ ), free of external constraints (mostly die-free and hanged by the electrodes), and subjected to much higher electric fields $\left(\mathrm{E}>50 \mathrm{~V} \cdot \mathrm{cm}^{-1}\right)$. Therefore, the denser green specimens in flash sintering provide continuous path for current flow. Consequently, the Joule heating at the contact points may be more prominent to aid sintering than spark and plasma at the inter-particle gaps. Hence, the capacitor element loses its importance in the present flash sintering model; therefore, only the resistor elements will be treated. The conductivity in such a granular system follows the random resistor lattices. ${ }^{15}$ Nevertheless, if spark and plasma are considered, the capacitor element should also be considered.

The following scenario of flash sintering is consistent with the experimentally observed electrical behavior. ${ }^{16,17}$ Surface conductivity in dielectrics increases with the increase in temperature, and is higher than that of the particles bulk conductivity. Therefore, at a certain temperature, when the compact can conduct enough current, Joule heating will become effective. Particles with different contact diameters will exhibit different contact resistances. The distribution of the contact resistances is expected to be normal or lognormal, similar to that of the particle size, since both are defined by the particle dimensions and geometry. Assuming a random distribution of the particles in the compact, the Joule heating will be more effective and preferable at the loci of the particle contacts, who exhibit maximum in the electric power generation.

Thus, at a given current density, some contacts will reach higher temperatures than others, due to the local Joule heating. The heat capacity of dielectrics generally increases with the increase in temperature, while their heat conductivity decreases. Consequently, the local heating at the preferred contacts assists local absorption of the generated heat, which, in turn, leads to an increase in the local temperature. Therefore, by means of energy consumption, the local heating at the preferred contacts has an autocatalytic character, i.e., repeated cycles of temperature increase leading to the increase in electrical conductivity $\rightarrow$ increase in current $\rightarrow$ increase in generated power $\rightarrow$ increase in temperature. Hence, for a constant voltage regime, where the current steadily increases, further heating would lead to local softening at the preferred contact points within the powder compact. This is also associated with the free molecular heat transfer regime, as will be discussed below. Due to the random distribution of the particles, one expects at a first glance that the first softening events will take place at random locations within the compact, providing a homogeneous symmetric system. However, as shown experimentally, ${ }^{18,19}$ and discussed theoretically, ${ }^{20}$ the current flow in FS is part of the electrochemical redox reaction; hence, the powder compact is an asymmetric electrochemical cell composed of the cathode and anode at its edges. Therefore, a pronounced reaction is reported to occur near the electrodes, either at the anode ${ }^{18}$ or at the cathode. ${ }^{19}$ The local softening events should start preferably at the anode side, where the electrons leave to the external circuit, and the ions reach from the cell center and propagate towards the cathode with a front. The width of such a front may be as large as the clusters (i.e., conducting backbones) formed within the compact. $^{21}$ The melt resistivity in most dielectrics is lower by two to four orders of magnitude relative to that of the solid bulk. $^{22,23}$ This drastic decrease in resistivity at the softened/ melted contact will increase the current (through it), as no contact resistor exists; to say that the contact resistor is invaded by a "fast-conducting" behavior (i.e., $\sigma_{\mathrm{m}} \gg \sigma_{p}$ ), when $\sigma_{m}$ and $\sigma_{p}$ are the melted/softened contact and the solid particle conductivities, respectively. ${ }^{21,24}$ Local softening decreases the local electric resistance; hence, energy consumption enhanced at other contacts with higher resistances. In the next step, the particle contacts with higher resistance than the former (now softened) will soften/melt. These softening events altogether occur within a fraction of a second, and the local melt will not solidify, unless a low-resistance percolating path between the electrodes formed. This arises from the self-regulating nature of the current flow, through the various "chains" of the random resistor network. Therefore, the local softening is in a temporary equilibrium with the solid. Invasion of the resistor contact-points with "fast-conductors," by softening, will proceed as a front in a general direction from the anode towards the cathode. The nature of such fronts in various percolating systems is fractal. The backbone is the set of contacts available for softening. Nevertheless, local softening and current percolation through contacts of the shortest distance between the two electrodes may form clusters with side branches, ending with resistors at their edges (no softening). These clusters constitute the percolating backbone and may contain 'trapped' sites (particles with non-melted contacts) within them. Therefore, the backbone may constitute a larger number of contacts than the number of (melted) bonds linearly connecting between the two electrodes. The backbone also has a fractal character with a fractal dimension $D_{B} \approx 1.74$ in $3 \mathrm{D}$ systems. ${ }^{11,25}$ Consequently, both dead ends and trapped sites contribute to densification if locally melted within the backbone, but do not contribute to current percolation. The clusters mentioned above are also associated with the particle rearrangement by softened layer capillary. The percolation threshold is reached when continuous invasion of an "infinite" cluster takes place and connects between the two electrodes. Therefore, invasive current percolation leads to a homogeneous densification of the compact; the only inhomogeneity 
may arise from the inhomogeneity of the green compact, and at the electrode/specimen interfaces, as reported, due to the electrochemical electrode asymmetry. ${ }^{19}$

The input Joule power generated at the particle contacts has first to be absorbed within the particles. Local surface softening is associated with high local temperature gradients, of the order of $2000 \mathrm{~K}$ to $3000 \mathrm{~K}$ between the solid particle and its melted/softened surface. ${ }^{26}$ At first glance, the typical thermal diffusivity of dielectrics in the bulk (i.e., $7.4 \times 10^{-7}$ $\mathrm{m}^{2} \cdot \mathrm{s}^{-1}$ for $\mathrm{Y}-\mathrm{TZP}^{27}$ ) is expected to lead to temperature homogenization within nanoseconds. However, such very high temperature gradients may survive if the Knudsen number (the ratio between the mean free path of the conducting gas molecule at the temperature and pressure of interest, divided by the particle radius) is large enough $(\mathrm{Kn} \geq 1)$. In this case, heat conduction regime shifts from the continuum regime to the free molecular regime. This change in the thermal behavior of the high temperature particle is associated with the formation of a surface layer (Langmuir) immediate to the particle surface, where the gas molecules are collisionless and do not conduct. ${ }^{28}$ Consequently, very high temperature gradients may evolve between the solid particle (center) and the particle melted/softened surface (contact), especially for nanoparticles at high temperatures and at ambient pressure. The analysis of the experimental data for nanocrystalline Y-TZP (Yttria-stabilized Tetragonal Zirconia Polycrystals) and micrometer-sized $\mathrm{SrTiO}_{3}$ from the literature ${ }^{16,29}$ reveal high values of Knudsen numbers, which correspond to free molecular thermal behavior at high flash sintering temperatures and the atmospheric pressure. ${ }^{28}$ Energy balance for the dissipated electric power revealed particle surface temperatures as high as $3387 \mathrm{~K}$ and $4489 \mathrm{~K}$ for Y-TZP and $\mathrm{SrTiO}_{3}$, respectively. ${ }^{26}$ Therefore, the melted layer may be a few atomic layers thick; hence, the surface melting be termed as surface softening. Recently, nanocrystalline ceramic compacts were rapidly heated either by rapid thermal annealing, ${ }^{30}$ or by rapid insertion into the furnace, ${ }^{31}$ where no electric field was applied. Densification levels close to those of flash sintering were recorded and may reveal the significant role of the temperature gradients in the flash sintering process.

The local softening at the particle contacts leads to a change in two physical properties, which have important implications for sintering. First, the melt specific volume increases and is higher than its previous solid volume. The volume increase of $10 \%$ to $20 \%$ often takes place during the softening of alumina, as a model system. ${ }^{32-34}$ The second universal behavior in dielectrics is the drastic increase by 2 to 4 orders of magnitude in the electric (or ionic) conductivity of the melt, compared to its crystalline counterpart. ${ }^{35,36}$ These relative values justify the application of the invasion percolative model with resistor (sites)__fast conductor" (bonds) elements at the percolation threshold, as made above.

For single-phase powder, full solubility between the solid and softened contacts exists. Therefore, simultaneously to the local softening, the melt fully wets the two adjacent particles at the contact area. The capillary force due to the softened meniscus and its pre-wetting front should impose strong attractive forces on the two adjacent particles, and lead to local rearrangement, sintering, and densification. The volume increase due to the melt aids the particle rearrangement, and leads to local densification. The level of the capillary forces, in the nanoparticle assembly, is of the order of the pressures typically used for hot-pressing (i.e., 5 to $50 \mathrm{MPa}$ ) of dielectric nanoparticles, and very effective for densification. Nevertheless, the mass relocation associated with the particle surface softening and its rearrangement is not a sufficient condition for full densification. This arises from the invasive nature of the percolating current (liquid) and the time needed for full wetting of the surfaces far from the contact points. Full wetting of the nanoparticle surfaces is a necessary condition for efficient densification as supported by stage III of flash, where the constant current regime has been kept for tens of seconds. ${ }^{37}$

A further increase in current or temperature will facilitate softening at contacts with slightly lower resistances than the first events. These consecutive softening events at random locations, but within a wide front, form rearranged clusters of grains (previously particles with single or polycrystalline nature) which vary in their size and shape. Therefore, the densification of the particles to dense clusters at the percolation threshold by self-organization via softened film capillary is one characteristic of this percolating system. It is worth noting that the impurity content and its segregation to the particle surfaces and grain interfaces may have a significant effect in triggering the local surface softening at temperatures lower than expected for the grain bulk. Dopants effects on the decrease in the onset flash voltage and temperature were demonstrated. ${ }^{9,38}$

Following the well-based percolation theory, the conductivity of the 3D percolating compact of particles modeled with $\sigma_{p}$ and $\sigma_{c}$ for the conductivities of particles and contacts, respectively. Generally, assuming a homogeneous system, the specific conductivity of the compact depends only on the ratio of these conductivities, and the fraction of the contacts through which the critical phenomena takes place. If no limit is put to the current (as often done for the sintering studies), there are no contacts forbidden for local softening. This sets the conditions for invasion percolation. At the percolation threshold the contacts soften/melt, i.e., invaded by softened surface, hence by "fast-conducting" behavior, relative to the particle conductivity $\left(\sigma_{c}\right.$ replaced by $\left.\sigma_{\mathrm{m}}\right)$. One may approach this critical point either from below or from the above. The specific conductivity of this system at DC, versus fraction of the softened/melted bonds, $p$, expressed by power-law relation if approached from below $^{39}$

$$
\sigma_{D C}=\sigma_{p}\left(p_{c}-p\right)^{-s} \quad \text { For } p<p_{c}
$$

where $p_{c}$ is the percolation threshold and $s$ is one of the critical exponents.

When the critical point approached from the above, the relation is

$$
\sigma_{D C}=\sigma_{m}\left(p-p_{c}\right)^{t} \quad \text { For } p>p_{c}
$$

Moreover, $t$ is the second critical exponent. 
However, at $p=p_{c}$ with both $\sigma_{p}$ and $\sigma_{c}$ having finite values, the specific conductivity is ${ }^{39}$

$$
\sigma_{D C} \cong \sigma_{p}^{u}\left(\sigma_{m}\right)^{1-u} \quad \text { At } p=p_{c},
$$

where $u$ is the third critical exponent. Since these behaviors relate to the same system, their exponents are interrelated via the homogenous function representation

$$
u=\frac{t}{s+t} .
$$

Following the above resistor (conductor) lattice, the critical exponents for the 3D system $\operatorname{are}^{11} s=0.7, t=1.70$ and $u=0.72$, with $p_{c}=0.247$.

The aspect of volume fraction of the softened nanoparticle surfaces was included in Equations (1)-(3) via the percolation threshold, i.e., when 0.247 of the total volume is "fast conducting" due to its softening, while the rest behaves as a regular solid conductor (dielectric). This threshold volume fraction is constant per percolating transport property and per its percolating fashion, since the granular system geometrically is self-similar.

Alumina was flash-sintered under the electric field of $1000 \mathrm{~V} \cdot \mathrm{cm}^{-1}$ in the furnace pre-heated to $1200^{\circ} \mathrm{C}$, using platinum electrodes. ${ }^{10}$ The corresponding "incubation time," i.e., the time from the current/voltage application up to the flash event at constant temperature, was $81 \mathrm{~s}$. The specific conductivity of $\mathrm{Al}_{2} \mathrm{O}_{3}$ compact versus fraction of the melted contacts (bonds) at $1200{ }^{\circ} \mathrm{C}$ was calculated using Equations (1)-(3), and the following values of the electric conductivities: $\sigma_{p}=2.8 \times 10^{-5}[\Omega \cdot \mathrm{cm}]^{-1}$ at $1200^{\circ} \mathrm{C},{ }^{10}$ and $\sigma_{m}=0.228$ $[\Omega \cdot \mathrm{cm}]^{-1}$ for alumina melt (at $2047^{\circ} \mathrm{C}$ ), ${ }^{35}$ and the results are shown in Figure 1. The curves in Figure 1 present the percolating behavior of the electric current, hence electric conductivity versus fraction $p$ of the softened/melted contacts. The $p$ axis implicitly expresses the process axis, i.e., increase in time, current, etc., in the compact. However, for comparison

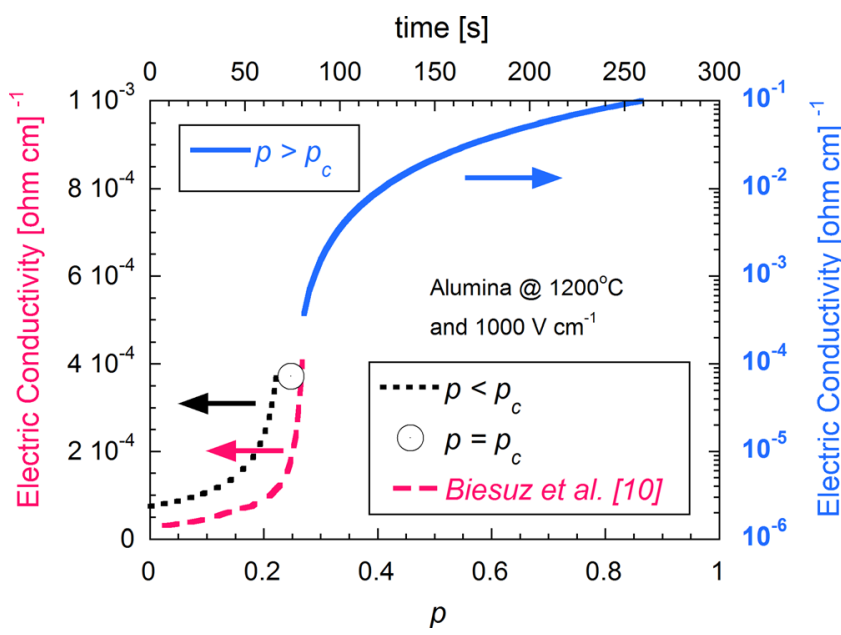

FIG. 1. Electric conductivity of $\alpha-\mathrm{Al}_{2} \mathrm{O}_{3}$ nanoparticles subjected to flash sintering at $1200{ }^{\circ} \mathrm{C}$ under $1000 \mathrm{~V} \cdot \mathrm{cm}^{-1}$ versus time (top x-axis) (Ref. 10), and the volume fraction of the melt $p$ (bottom $\mathrm{x}$-axis). The experimental (red dashed-curve) coincides with the calculated (dotted black-curve) from the percolation model. The flash "incubation time" of $81 \mathrm{~s}$ was adjusted to the percolation threshold at $p_{c}=0.247$ (open circle). with the experimental results, ${ }^{10}$ the $p$ axis should be calibrated such that to explicitly expresses the time up to the flash event. The observed "nucleation" of the flash event ${ }^{10,40}$ is manifestation of the current percolation threshold in the dielectric granular compact. The so-called "incubation time" to the non-linear electrical behavior (flash event) ${ }^{10,41}$ is the time needed for local softening of the nano-particle contacts and wetting, to form a percolative pass for the electric current between the electrodes. Therefore, the percolation threshold $p_{c}=0.247$ (bottom $\mathrm{x}$-axis in Figure 1) was equated to the "incubation time" of $81 \mathrm{~s}$ (Ref. 10) for the time axis (top x-axis in Figure 1). The experimentally measured conductivity (red dashed-curve in Figure 1) is very close to that calculated (black dotted-curve in Figure 1) using the percolative model, and plotting them in a linear scale (left Y-axis). This confirms the flash process as an electric current percolation phenomenon. The open circle in Figure 1 represents the actual conductivity value at the percolation threshold $p=p_{c}=0.247$. The blue solid-curve in Figure 1 shows the state of granular conductivity in the absence of current limit. Therefore, the change from the voltage control mode to the current control mode (the so-called stage I to stage II) ${ }^{37}$ in the flash experiments actually preserves the transient and locally formed softened/melted layers at the contact points, and enables their propagation throughout the specimen, as long as non-rigid network of particles exist.

Generally, the details of the calculated curves in Figure 1 may vary with the particle size, pore size, as well as the specimen dimensions and heating rates, since these geometric and process parameter values modify the pre-factors in Equations (1)-(3). Nevertheless, the percolative character remains unchanged according to a universal behavior of the system discussed above.

Recently, a similar percolation model was introduced for rapid densification of dielectric oxide using flash assisted microwave sintering. ${ }^{42}$ The remnants of a relatively thick softened layer were observed at the grain boundaries of $\mathrm{Yb} / \mathrm{La}$ doped $\mathrm{Y}_{2} \mathrm{O}_{3}$ subjected to flash microwave sintering. However, the rapid densification was related to the thermal stresses originating from the presence of solid grain-melt grain boundary two-phase assemblage, rather than the capillary force caused by the particle surface softening at the contacts. As was mentioned earlier, a few atomic layers softened/melted nanoparticle surfaces have a transient character; hence, melted residue in pure oxides can barely be observed. Nevertheless, melting at the microcrystal surfaces or at the grain boundaries where melting led to compositional ${ }^{43,44}$ or morphological ${ }^{2,45-47}$ changes during the SPS/flash sintering were reported.

Finally, a recent grain size analysis of the dog boneshape flash sintered spinel specimen along its length clearly revealed enhanced grain growth at the positive electrode. ${ }^{48}$ This further supports the electrochemical nature of the specimens subjected to flash sintering and their corresponding percolative character as granular dielectrics.

\section{CONCLUSIONS}

Flash sintering belongs to the framework of the critical phenomena, where electric conductivity suddenly changes 
by several orders of magnitude, at critical values of temperature and applied electric field. The electrical behavior of the dielectric powder compact with the increase in temperature, current, voltage, and duration follows that of random resistor array with a percolating character. The experimental conductivity, during the flash sintering of alumina, was in agreement with the calculated conductivity using the percolative model. The asymmetric nature of the compact as an electrochemical cell subjected to flash sintering, and the preferred local surface softening/melting at the propagating front, characterizes the invasive percolation nature of the system. The fractal nature of the propagating front, and the conducting backbone, leads to the self-organizing character of the dielectric nanoparticles, aided by attractive capillary forces, due to the softened/melted surface at the contacts.

${ }^{1}$ R. Chaim, J. Mater. Sci. 48, 502 (2013).

${ }^{2}$ T. Saunders, S. Grasso, and M. J. Reece, J. Eur. Ceram. Soc. 35, 871 (2015).

${ }^{3}$ R. Marder, C. Estournès, G. Chevallier, and R. Chaim, Scr. Mater. 82, 57 (2014).

${ }^{4}$ Z. Zhang, Z. Liu, J. Lu, X. Shen, F. Wang, and Y. Wang, Scr. Mater. 81, 56 (2014).

${ }^{5}$ R. Marder, C. Estournès, G. Chevallier, and R. Chaim, J. Eur. Ceram. Soc. 35, 211 (2015).

${ }^{6}$ W. Ji, S. S. Rehman, W. Wang, H. Wang, Y. Wang, J. Zhang, F. Zhang, and Z. Fu, Sci. Rep. 5, 15827 (2015).

${ }^{7}$ R. Chaim, Scr. Mater. 115, 84 (2016).

${ }^{8}$ M. Yu, S. Grasso, R. Mckinnon, T. Saunders, and M. J. Reece, Adv. Appl. Ceram. 116, 24 (2017).

${ }^{9}$ M. Cologna, J. S. C. Francis, and R. Raj, J. Eur. Ceram. Soc. 31, 2827 (2011).

${ }^{10}$ M. Biesuz, P. Luchi, A. Quaranta, and V. M. Sglavo, J. Appl. Phys. 120, 145107 (2016).

${ }^{11}$ J. F. Gouyet, Physics and Fractal Structures (Masson, Paris and Springer, New York, 1996).

${ }^{12}$ B. Kozlov and M. Lagues, Physica A 389, 5339 (2010).

${ }^{13}$ J. Gonzalez-Julian and O. Guillon, J. Am. Ceram. Soc. 98, 2018 (2015).

${ }^{14}$ R. Marder, C. Estournès, G. Chevallier, and R. Chaim, J. Mater. Sci. 50, 4636 (2015).

${ }^{15}$ J. P. Straley, J. Phys. C: Solid State Phys. 15, 2333 (1982).

${ }^{16}$ R. Raj, J. Eur. Ceram. Soc. 32, 2293 (2012).

${ }^{17}$ R. I. Todd, E. Zapata-Solvas, R. S. Bonilla, T. Sneddon, and P. R. Wilshaw, J. Eur. Ceram. Soc. 35, 1865 (2015).

${ }^{18}$ Y. Zhang, J. I. Jung, and J. Luo, Acta Mater. 94, 87 (2015).

${ }^{19}$ W. Qin, H. Majidi, J. Yun, and K. van Benthem, J. Am. Ceram. Soc. 99, 2253 (2016).
${ }^{20}$ L. B. Caliman, R. Bouchet, D. Gouvea, P. Soudant, and M. C. Steil, J. Eur. Ceram. Soc. 36, 1253 (2016).

${ }^{21}$ E. Clément, C. Baudet, E. Guyon, and J. P. Hulin, J. Phys. D: Appl. Phys. 20, 608 (1987)

${ }^{22}$ A. L. Leu, S. M. Ma, and H. Eyring, Proc. Natl. Acad. Sci. U.S.A. 72, 1026 (1975).

${ }^{23}$ V. I. Lakomsky, Part 3: Oxide Cathodes for the Electric Arc, in Welding and Surface Reviews, edited by B. E. Paton (Harwood Academic Publishers, Reading, UK, 2000), Vol. 13, pp. 74-90.

${ }^{24}$ D. Wilkinson and J. F. Willemsen, J. Phys. A 16, 3365 (1983).

${ }^{25} \mathrm{C}$. Li and T. W. Chou, Int. J. Mod. Phys. C 20, 423 (2009).

${ }^{26}$ R. Chaim, Materials 10, 179 (2017).

${ }^{27}$ J. M. Lebrun, S. K. Jha, S. J. McCormack, W. M. Kriven, and R. Raj, J. Am. Ceram. Soc. 99, 3429 (2016).

${ }^{28}$ F. Liu, K. J. Daun, D. R. Snelling, and G. J. Smallwood, Appl. Phys. B 83, 355 (2006)

${ }^{29}$ N. Shomrat, S. Baltianski, E. Dor, and Y. Tsur, J. Eur. Ceram. Soc. 37, 179 (2017).

${ }^{30}$ Y. Zhang, J. Nie, J. M. Chan, and J. Luo, Acta Mater. 125, 465 (2017).

${ }^{31}$ W. Ji, B. Parker, S. Falco, Y. Y. Zhang, Z. Y. Fu, and R. I. Todd, J. Eur. Ceram. Soc. 37, 2547 (2017).

${ }^{32}$ A. D. Kirshenbaum and J. A. Cahill, J. Inorg. Nucl. Chem. 14, 283 (1960).

${ }^{33}$ F. T. Wallenberger, N. E. Weston, K. Motzfeldt, and D. G. Swartzfager, J. Am. Ceram. Soc. 75, 629 (1992)

${ }^{34}$ J. M. Lihrmann and J. S. Haggerty, J. Am. Ceram. Soc. 68, 81 (1985).

${ }^{35}$ I. Pozniak, A. Pechenkov, and A. Shatunov, "Electrical conductivity measurement of oxide melts," in Modeling for Materials Processing (International Scientific Colloquium, Riga, 2006).

${ }^{36}$ J. Pappis and W. D. Kingery, J. Am. Ceram. Soc. 44, 459 (1961).

${ }^{37}$ S. K. Jha, K. Terauds, J. M. Lebrun, and R. Raj, J. Ceram. Soc. Jpn. 124, 283 (2016).

${ }^{38}$ R. Raj, J. Am. Ceram. Soc. 99, 3226 (2016).

${ }^{39}$ J. P. Straley, Phys. Rev. B 15, 5733 (1977).

${ }^{40}$ K. S. Naik, V. M. Sglavo, and R. Raj, J. Eur. Ceram. Soc. 34, 4063 (2014).

${ }^{41}$ E. Bichaud, J. M. Chaix, C. Carry, M. Kleitz, and M. C. Steil, J. Eur. Ceram. Soc. 35, 2587 (2015)

${ }^{42}$ Y. V. Bykov, S. V. Egorov, A. G. Eremeev, V. V. Kholoptsev, K. I. Rybakov, and A. A. Sorokin, J. Am. Ceram. Soc. 98, 3518 (2015).

${ }^{43}$ J. Du, A. P. Sanders, V. Jindal, and K. S. Ravi Chandran, Scr. Mater. 123, 95 (2016).

${ }^{44}$ H. Yoshida, A. Uehashi, T. Tokunaga, K. Sasaki, and T. Yamamoto, J. Ceram. Soc. Jpn. 124, 388 (2016).

${ }^{45}$ E. Zapata-Solvas, D. Gomez-Garcia, A. Dominguez-Rodriguez, and R. I. Todd, Sci. Rep. 5, 8513 (2015).

${ }^{46}$ Y. J. Wu, J. Li, X. M. Chen, and K. Kakegawa, Mater. Sci. Eng. A 527, 5157 (2010).

${ }^{47}$ K. Sun, J. Zhang, T. Jiang, J. Qiao, W. Sun, D. Rooney, and Z. Wang, Electrochim. Acta 196, 487 (2016).

${ }^{48}$ H. Yoshida, P. Biswas, R. Johnson, and M. K. Mohan, J. Am. Ceram. Soc. 100, 554 (2017). 